\title{
Disociación del liner acetabular tras una artroplastia total de cadera
}

\author{
Acetabular liner disassociation after total hip arthroplasty
}

\author{
René Ochoa Cázares*, Alberto Carlos Cervantes Ordoñez, ${ }^{\ddagger}$ René Gabriel Narváez Corona ${ }^{\S}$
}

\section{Resumen}

La disociación del liner acetabular es una complicación rara de la artroplastia total de cadera; sin embargo, su aparición pone en riesgo la integridad y la función de la prótesis. Se debe tener una alta sospecha clínica en pacientes con dolor tras una función protésica adecuada y que presentan un chirrido audible. Radiográficamente puede pasar desapercibida, ya que no suele ser tan apreciable como cuando hay una luxación protésica. Sus principales causas asociadas son el diseño del componente, la mala posición debido a una inclinación y anteversión incorrectas, y el pinzamiento del componente. El diseño modular del componente acetabular inevitablemente es un riesgo para este tipo de evento, por lo que siempre que se utilice un liner acetabular se debe comprobar que esté bien anclado a la copa acetabular durante su colocación y verificar que no haya tejido interpuesto antes de ser impactado, además de una colocación correcta de componentes.

Palabras clave: Disociación de liner, artroplastia de cadera.

\section{INTRODUCCIÓN}

La artroplastia total de cadera es un procedimiento común en la práctica ortopédica. El componente acetabular no cementado requiere de una interfaz de polietileno llamada liner acetabular, el cual, en conjunto con la copa acetabu-

* Profesor del Curso de Artroscopia y Reconstrucción Articular. Hospital Ángeles Pedregal. Facultad Mexicana de Medicina, Universidad La Salle, Campus México.

* Ortopedista. Hospital Ángeles Metropolitano, Ciudad de México.

$\S$ Alumno de Artroscopia y Reconstrucción Articular. Hospital Ángeles Pedregal. Facultad Mexicana de Medicina, Universidad La Salle, Campus México.

Correspondencia:

René Gabriel Narváez Corona

narvaezrg87@gmail.com

Aceptado: 01-08-2019.

www.medigraphic.com/actamedica

\section{Abstract}

Dissociation of the acetabular liner is a rare complication after a total hip arthroplasty. However, when it happens, the integrity and functionality of the prosthesis is at risk. The clinician must be aware of this possible complication in patients that report a sudden onset of pain in a previously functional prosthesis, followed by an audible squeaking. Radiographs may be unnoticed because the changes usually not as remarkable as in a THA dislocation. The most important causes are the component design, malposition and impingement. A modular component is inevitably a risk for these events, and one always should check that the locking mechanism works and avoid interposed tissue before its impaction, and a correct anteversion and inclination of the acetabular component.

Keywords: Liner dissociation, hip arthroplasty.

lar, reciben la cabeza femoral, generalmente ensamblada a un vástago para formar la nueva articulación de cadera. Su capacidad modular permite diferentes combinaciones de pares de fricción, cada una con sus ventajas y desventajas. Sin embargo, este ensamble entre el liner y la copa acetabular puede aflojarse en muy raras ocasiones, lo cual se ha descrito en alrededor de $0.04 \%$ de los casos. ${ }^{1,2} \mathrm{~A}$ continuación, presentamos el caso de una disociación de liner acetabular tratada en el Hospital Ángeles Metropolitano a un mes de realizarse una artroplastia total de cadera.

\section{CASO CLÍNICO}

Se presenta el caso de un paciente masculino de 75 años, postoperado de artroplastia total de cadera izquierda el día 9 de abril de 2019. Inició dos semanas previas a su consulta el día 7 de mayo de 2019 con dolor de cadera izquierda, el cual estuvo localizado en la región inguinal, sin irradiaciones y acompañado de una sensación vibratoria y 
auditiva de chirrido al movilizar la cadera que es también perceptible por el familiar y el médico tratante.

Previamente al manejo protésico, el paciente presentaba una limitación importante de los arcos de movilidad, los cuales estaban acompañados de dolor, por lo que se le dificultaba de manera importante las actividades diarias y la marcha. Radiográficamente el paciente presentaba pinzamiento articular, esclerosis subcondral, osteofitos y deformidad con pérdida de la esfericidad de la cabeza femoral, lo que la clasifica como una artrosis tipo IV de la clasificación de Tönnis. En su primer acto quirúrgico se colocó una prótesis total de cadera no cementada Synergy (Smith \& Nephew, Memphis, TN) con vástago femoral 17, cabeza femoral de oxinium 36, y copa acetabular R3 (Smith \& Nephew, Memphis, TN) 58 con liner acetabular 58 con ceja posterior.

Al momento de su valoración se encontró realizando marcha asistida con andadera, y presentó dolor aun con apoyo y al realizar movimientos. Terminó las sesiones de fisioterapia. Se realizó valoración radiográfica de proyección anteroposterior de pelvis en la que se observa pérdida de la centralización de cabeza femoral protésica, la cual está lateralizada y aún contenida en la copa acetabular. Se realizó el diagnóstico de disociación de liner acetabular de artroplastia total de cadera (ATC) izquierda.

\section{TÉCNICA QUIRÚRGICA}

Se programó al paciente para revisión del componente acetabular al día siguiente, en el que se efectuó abordaje posterolateral sobre el abordaje previo, se disecó por planos, se incidió el tensor de la fascia lata y las fibras proximales del glúteo mayor, y se refirieron y desinsertaron los músculos rotadores externos de piramidal a cuadrado femoral; se realizó la capsulotomía posterior y se observaren las rayaduras en la superficie de la cabeza femoral de oxinium, y horizontalización y protrusión medial de liner acetabular. Se extrajo el liner y se valoró la integridad de éste, evidenciando aplanamiento de algunas de las lengüetas antirrotacionales del componente del liner.

Se revisó la posición de la copa acetabular, la cual se encontró con una inclinación de $45^{\circ}$ y anteversión de $10^{\circ}$, se limpió el tejido interpuesto y se hizo el cambio por el liner con ceja posterior de medida 58, y cabeza $36+3$. Se realizaron las pruebas para verificar la implantación adecuada del liner, sin lograr su desanclaje. Se redujeron los componentes y se realizaron las pruebas de estabilidad; se verificó nuevamente la posición de los componentes con control radiográfico satisfactorio. Se reparó la cápsula articular, los rotadores externos y se cerraron por planos hasta llegar a la piel. El paciente fue dado de alta dos días

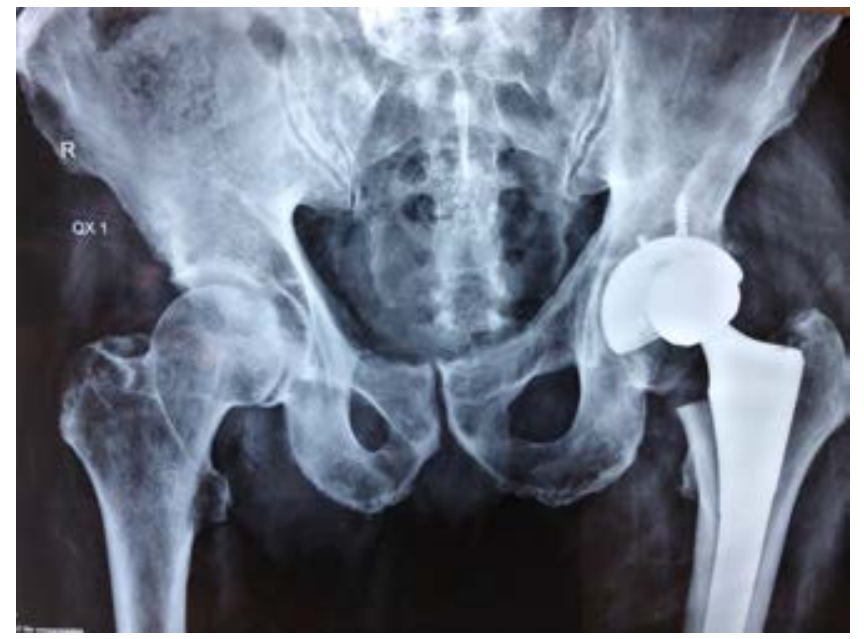

Figura 1: Migración superior y lateral de la cabeza femoral, sin salir de los límites de la copa acetabular.

después y fue valorado el día 11 de mayo de 2019, presentando marcha en andadera sin dificultad, la herida bien cerrada sin sangrado o exudado y negando dolor con el apoyo o sensación de chirrido nuevamente.

\section{DISCUSIÓN}

La artroplastia total de cadera es un procedimiento común, que ha sido descrito por la revista The Lancet como la cirugía del siglo, debido a la calidad de vida que ofrece dicho procedimiento y a los avances que se han logrado en su desarrollo. ${ }^{3}$ Sin embargo, existen complicaciones que, debido a su rara presentación, no se ha podido establecer de forma clara cómo es que éstas ocurren, como es el caso de la disociación del liner acetabular.

Esta complicación tiene una incidencia del $0.04 \%,{ }^{1}$ aunque se han publicado estudios más recientes en los que se estima una frecuencia entre 0.17 y $0.82 \% .^{4}$ Se ha asociado con falla en el mecanismo de cierre entre la copa acetabular y el liner, y parece estar más asociada con el liner de polietileno que de cerámica, por diferencias en el mecanismo de cierre dadas por el diseño geométrico y las propiedades del material empleado para el liner, el cual está sometido a condiciones adversas tales como el torque y pinzamiento. Los diseños pueden presentar mecanismos antirrotacionales a manera de lengüetas, los cuales se ha visto que fallan. Además, los casos de falla tardía se suelen asociar con una mala colocación de los implantes. ${ }^{1}$

Los síntomas más comunes en esta complicación suelen ser el dolor súbito en una prótesis que funcionaba adecuadamente, acompañado de una sensación de chirrido o 


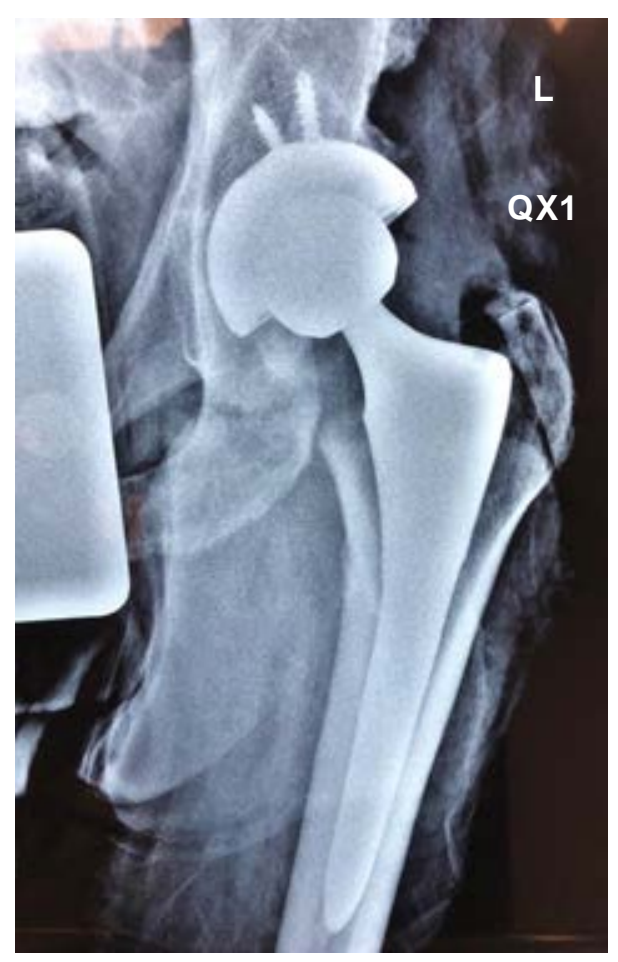

Figura 2:

Tras la revisión de la artroplastia total de cadera (ATC), se observa centralización de la cabeza femoral.

chasquidos en la articulación, perceptibles por el paciente y en ocasiones por quienes lo rodean. No es esperable encontrar los hallazgos de una luxación protésica, debido a que la cabeza sigue estando contenida por el componente acetabular; sin embargo, con los movimientos de cadera, el metal de la cabeza femoral protésica se talla contra el metal del componente acetabular, lo que produce un chirrido audible. ${ }^{1}$

Los estudios de imagen previos suelen ser de gran utilidad para evidenciar una migración lateral y superior de la cabeza femoral, la cual continúa siendo contenida por el componente acetabular, pero sin encontrarse centralizada. ${ }^{1}$

Entre los diseños protésicos más asociados con la disociación se encuentra el sistema Pinnacle de DePuy y el Harris-Galante de Zimmer. ${ }^{2,4}$ Se ha reportado un estudio en el que se incluyeron 23 casos con disociación atraumática en un periodo de 48 meses desde la cirugía, entre 2001 y 2013, debido a la disrupción de las lengüetas antirrotacionales del liner, las cuales se encuentran alternadas para los espacios disponibles en la copa. Gray y colaboradores asocian el riesgo de falla, en este diseño, a la posición inadecuada y pinzamiento exagerado en los dispositivos que cambian la orientación del liner, además de que sobresale de la copa acetabular en este diseño. Otro factor de riesgo a considerar es que los tornillos de la copa acetabular se encuentren protruidos. Se ha iden- tificado también que tras la introducción del polietileno de alta densidad de enlaces cruzados puede presentarse un incremento en la ruptura de las lengüetas antirrotacionales, ya que el polietileno de enlaces cruzados es más resistente al desgaste, pero con menor fuerza mecánica, por lo que se reblandece con el tiempo. ${ }^{2,4}$

El pinzamiento del cuello femoral protésico contra el liner, en combinación con una posición inadecuada o liners con cambios en la configuración de su superficie (offset o cambio de orientación) son también considerables en casos de disociación de liner, así como en casos de luxaciones protésicas. ${ }^{4}$

El uso de cabezas femorales protésicas de oxinium ha permitido que las artroplastias tengan la efectividad de la superficie de un cerámico, con lo que se reduce de manera importante el desgaste y la producción de micropartículas sin el riesgo de fracturar la cabeza a partir de un material hecho de óxido de zirconio. ${ }^{5}$

Otro reporte de caso comenta la progresión de metalosis en una paciente tras tres años de haberse sometido a una artroplastia total de cadera con una cabeza de oxinium, en la cual se describe que ésta puede presentar un desgaste acelerado con falla catastrófica del implante. Al ser un material más blando que la copa acetabular, la cabeza sufre una deformidad plástica importante cuando se pone en contacto con la copa, debido a que su sustrato es más blando. En estos casos los niveles de cobalto y cromo no son de utilidad para valorar la metalosis, debido a que la cabeza femoral está hecha de zirconio. ${ }^{5}$

La metalosis es la precipitación de partículas metálicas a la piel y tejidos producida por la corrosión y desgaste de la superficie articular protésica. Generalmente se limita a la cápsula y tejidos periprotésicos. Se debe considerar la metalosis como secundaria a falla de material en disociaciones metal-metal en pacientes que presenten decoloración cutánea acompañada de una artroplastia dolorosa y poco funcional. ${ }^{6}$

Se recomienda realizar una revisión del mecanismo de cierre del liner tratando de extraerlo y una inspección visual de toda la periferia del liner, previamente al cierre de la herida quirúrgica, y que los tornillos no queden protruidos. $^{2}$ Si se observa que la copa acetabular se encuentra pulida o con mala posición es recomendable hacer un recambio de este componente a expensas de presentar nuevamente disociaciones del liner. ${ }^{4}$ También se recomienda hacer seguimiento cardiaco, neurológico y dermatológico en pacientes que hayan presentado casos de metalosis, así como niveles séricos de cromo y cobalto en visitas subsecuentes, dependiendo del implante utilizado y, finalmente, considerar los implantes constreñidos en revisiones de componentes. ${ }^{5}$ 


\section{CONCLUSIÓN}

La disociación de un liner acetabular puede ser un evento catastrófico para el pronóstico funcional del paciente postoperado de prótesis de cadera. Deben procurarse cuidados al momento de la colocación de la prótesis para evitar que esta situación se presente, siempre verificando la implantación adecuada y que el mecanismo de cierre del liner funcione antes del cierre de la herida. En ocasiones, con los cuidados adecuados, puede no ser suficiente para evitar esta complicación, ya que en este caso los componentes presentaban una alineación adecuada y no se evidenció material interpuesto entre los componentes. Sin embargo, el diseño protésico puede presentar fallas, lo que produce, como ocurrió en este caso, el aplanamiento de las lengüetas antirrotacionales del liner acetabular.

\section{REFERENCIAS}

1. O'Neill CKJ, Napier RJ, Diamond OJ, O'Brien S, Beverland DE. Acetabular Liner Dissociation following Total Hip Arthroplasty: A Rare but Serious Complication That May Be Easily Misinterpreted in the Emergency Department. Care Rep Emerg Med. 2015; 2015: 1-4.

2. Yun A, Koli EN, Moreland J, lorio R, Tilzey JF, Mesko JW, Froimson M. Polyethylene Liner Dissociation Is a Complication of the DePuy Pinnacle Cup: A Report of 23 Cases. Clin Orthop Relat Res. 2015; 474: 441-446.

3. Learmonth ID, Young C, Rorabeck C. The operation of the century: total hip replacement. Lancet. 2007; 370: 1508-1519.

4. Kagan R, Anderson MB, Peters C, Pelt C, Gililland J. Pinnacle polyethylene liner dissociation: a report of 3 cases. Arthroplast Today. 2018; 4: 441-446.

5. Zou AH, Novikov D, Feng JE, Anoushiravani AA, Schwarzkopf R, Vigdorchik JM. Liner dissociation leading to catastrophic failure of an Oxinium femoral head. Arthroplast Today. 2019; 5: 21-25.

6. Sporer SM, Chalmers PN. Cutaneous Manifestation of Metallosis in a Metal-on-Metal Total Hip Arthroplasty After Acetabular Liner Dissociation. J Arthroplasty. 2012; 27: 1580.e13-1580.e16. 